\title{
Stellar Population of the Decoupled Nucleus in M31
}

\author{
O.K. Sil'chenko \\ Sternberg Astronomical Institute, Moscow, 119899 Russia \\ Isaac Newton Institute, Chile, Moscow Branch
}

\author{
A.N. Burenkov ${ }^{1}$ and V.V. Vlasyuk \\ Special Astrophysical Observatory, Nizhnij Arkhyz, 357147 Russia
}

\begin{abstract}
Spectral and imaging results for the central region of M31 are presented. An analysis of absorption-index radial profiles involving $\mathrm{Mg}, \mathrm{Fe}$, and $\mathrm{Ca}$ lines reveals that the unresolved core of M31 is distinct from the inner bulge in having a higher metallicity; of the two nuclei of $\mathrm{M} 31$, it is the fainter in visible light and is located exactly in the dynamical center of the galaxy (and dynamically decoupled) which is chemically distinct. By including the Balmer absorption line $H \beta$ in the analysis, we have disentangled metallicity and age effects; a stellar age difference by a factor of three is found between the nucleus and the bulge, the nucleus being younger. A morphological analysis of CCD images has shown the presence of an inner stellar-gaseous disk with a radius of some $80 \mathrm{pc}$ which is embedded into the bulge.
\end{abstract}

\section{Introduction}

During several years we have searched for chemically distinct stellar nuclei in early-type spiral galaxies, that is, for the nuclei whose stellar population is prominently more metal-rich than the stellar population of the nearby bulge. We have already found such nuclei, mainly unresolved, in some nearby galaxies, such as NGC 2841 (Sil'chenko et al. 1997), NGC 4826 (Sil'chenko 1996), and others. The most nearby early-type spiral, M31, possesses a chemically distinct nucleus too.

The chemical distinctness of the nucleus of M31 was first noticed some decades ago. A brief list of papers concerning this topic is presented in Table 1. Unfortunately, most of these works were performed before modern CCDs became in common use. The only exception is the work of Davidge (1997); but he does not touch the nucleus, being concentrated on gradients of absorptionline equivalent widths in the inner bulge. Another, even more recent paper of Davidge et al. (1997) treats the properties of the stellar population in the nucleus of M31, based completely on photometric data; their CM diagrams for the brightest stars, being constructed under very good seeing conditions in three

${ }^{1}$ RGO Astronomy Data Centre, Guest Investigator 
narrow concentric rings inside $R=4.0^{\prime \prime}$, have allowed detection of a noticeable increase in mean stellar age with radius. Meantime the metallicity estimates remain rather uncertain. In general, though the existence of a metallicity difference between the nucleus and the bulge of M31 has been established long ago, up to now quantitative estimates ranged from 0.2 dex to more than an order of magnitude.

Table 1. Papers on chemical decoupling of the nucleus of M31.

\begin{tabular}{lcc} 
Paper & Method/Line or Colour & Metallicity difference \\
\hline McClure 1969 & Narrow-band photometry, CN $\lambda 4150$ & \\
Joly \& Andrillat 1973 & Spectroscopy, MgI & 1.5 dex \\
Joly \& Andrillat 1973 & Spectroscopy, CaIIK & 0.6 dex \\
Spinrad \& Liebert 1975 & Broad-band photometry, $U-V$ & 0.2 dex \\
Morton \& Andereck 1976 & Spectroscopy, CaIIK & 0.6 dex \\
Cohen 1979 & Spectroscopy, MgI, NaID & 0.6 dex \\
Bica et al. 1990 & Spectroscopy, MgI, CaIIK & 0.2 dex
\end{tabular}

The unresolved nucleus of M31 is also known to be dynamically decoupled. Among the basic works on this topic we can mention long-slit spectroscopy of Kormendy (1988) and Dressler \& Richstone (1988) and the complex bi-dimensional study of Bacon et al. (1994). After the HST high-resolution imaging it has become clear that M31 has two nuclei (Lauer et al. 1993). The optically fainter nucleus is located in the center of isophotes, and the brighter one is shifted by 0 '.5. It is the fainter nucleus which is at the dynamical center. Its dynamical decoupling together with a high mass-to-luminosity ratio imply it to be a supermassive black hole; but there is also some evidence for the existence of a dynamically decoupled compact stellar disk in the center of M31: Kormendy (1988) found a decrease of stellar velocity dispersion inside a radius of $4^{\prime \prime}$, and a popular model of Tremaine (1995) includes a thick eccentric elliptical stellar disk.

We have undertaken a long-slit spectral investigation of the stellar population properties in the nucleus and in the inner bulge of M31. By calculating different Lick-system absorption-line indices, we have now been able to compare both the mean metallicity and the mean age of stars and to determine in this way a sequence of star formation epochs. A separate, very interesting task was to identify a chemically distinct entity within one of Andromeda's two known nuclei. For this purpose we have used data from the La Palma Archive.

\section{Observations}

In this work we use the following observational data:

- Our long-slit spectroscopy: $1 \mathrm{~m}$ telescope of the Special Astrophysical Observatory, spectrograph UAGS+CCD $1040 \times 1160$, slit width 3 "'2, seeing $4^{\prime \prime}$, scale along the slit $1^{\prime \prime} 54$, spectral range $3900-5700 \AA$, resolution $4 \AA$. Exposures: September $11,1996, P A=35^{\circ}, 80^{\circ}$, and $125^{\circ}$, each 40 minutes; September 12 , 1996, $P A=155^{\circ}$ and $-10^{\circ}$, each 30 minutes.

- La Palma long-slit spectroscopy: 4.2m WHT, spectrograph ISIS+CCD $800 \times$ 1180 , slit width $0 ! 7$, seeing $0 ! 9$, scale along the slit $0 ! 34$, spectral range 3800 


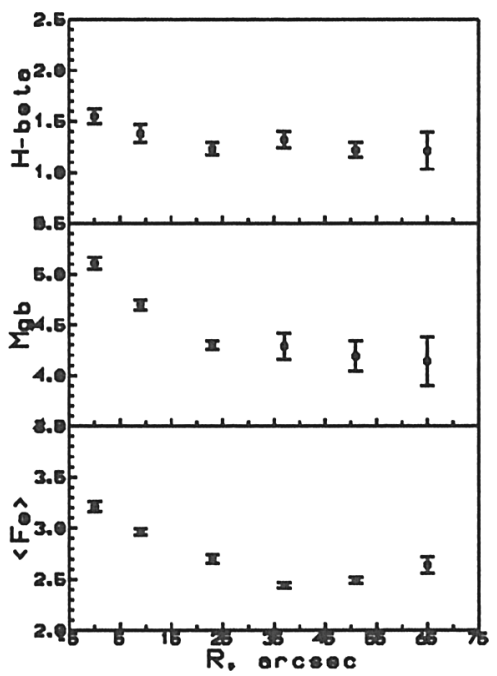

Figure 1. Radial profiles of absorption-line indices $H \beta, \mathrm{Mgb}$, and $<\mathrm{Fe}>$ averaged over radius intervals of $14^{\prime \prime}$ and over five different position angles.

$4700 \AA$, resolution $2 \AA$. Exposure: September 19, 1991, $P A=55^{\circ}, 90$ minutes. - La Palma photometry: $1 \mathrm{~m}$ JKT, CCD RCA $320 \times 512$, scale 0 ' 41 per pix, seeing 1".5. Exposures: November 17, 1990, $B, V, R$, each $500 \mathrm{~s}$, and $I, 1000 \mathrm{~s}$. Reduction of the data has been made by using the software of Vlasyuk (1993).

\section{Results}

From our five cross-sections of M31 obtained at the $1 \mathrm{~m}$ telescope of SAO RAS we have calculated index profiles for $H \beta, \mathrm{Mgb}, \mathrm{Fe} 5270$ and Fe5335 up to $70^{\prime \prime}$ from the center. The two iron indices were merged into $<\mathrm{Fe}>\equiv(\mathrm{Fe} 5270+\mathrm{Fe} 5355) / 2$. The results are presented in Fig. 1.

In accordance with numerous results of earlier investigations, the center of M31 is distinct by having the higher equivalent widths of magnesium and iron lines. The measurements at $r=9^{\prime \prime}$ may be affected by seeing. At larger distances from the center radial index gradients, if they exist, are negligible with respect to the index changes between the nucleus and the bulge. The mean index values in the bulge in the radius range $23^{\prime \prime}-65^{\prime \prime}$ are $\mathrm{Mgb}$ (bulge) $=4.25 \pm 0.02$ and $<\mathrm{Fe}\rangle$ (bulge) $=2.56 \pm 0.01$. In the nucleus $\mathrm{Mgb}($ nuc $)=5.11 \pm 0.06$ and $<\mathrm{Fe}>$ (nuc) $=3.21 \pm 0.05$. Therefore, the differences between the nucleus and the bulge are $\Delta \mathrm{Mgb}=0.86 \pm 0.10$ and $\Delta<\mathrm{Fe}>=0.65 \pm 0.06$. Application of models of Worthey (1994) for an old stellar population under the assumption of equal bulge and nucleus ages and of a solar ratio $\mathrm{Mg} / \mathrm{Fe}$ gives an estimate of metallicity difference, $0.42 \pm 0.05 \mathrm{dex}$, the same for magnesium and iron. But are the assumptions of equal ages for the nucleus and bulge stellar populations and of solar magnesium-to-iron ratio valid in this particular case? 

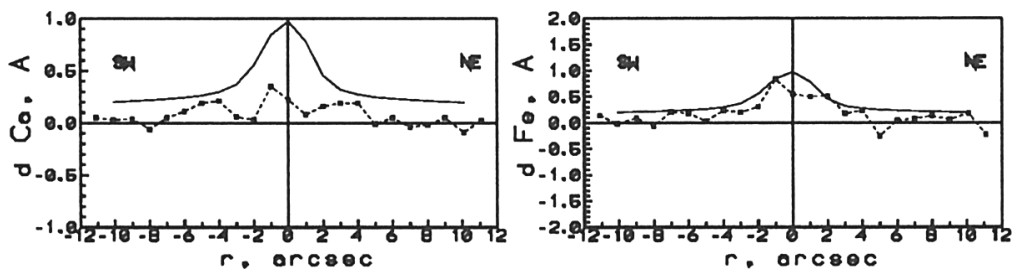

Figure 2. Radial profiles of $(\Delta \mathrm{Ca} 4227+\Delta \mathrm{Ca} 4455) / 2$ and $(\Delta \mathrm{Fe} 4383+$ $\Delta \mathrm{Fe} 4531) / 2$ indices after removing linear radial trends; the solid line shows the continuum profile near $\lambda \approx 4400 \AA$, the positions of the brightest continuum points are marked by vertical lines.

If the nucleus is chemically decoupled, it would be natural to suggest that the epochs of basic star formation in the nucleus and in the bulge are different. So the mean ages of the stellar populations in the nucleus and in the bulge are expected to be different. Recently Tantalo et al. (1998) have presented new model calculations allowing to determine differences in metallicity, age, and magnesium-to-iron ratio from the $H \beta, \mathrm{Mg}_{2}$, and $<\mathrm{Fe}>$ differences. We have taken the index differences between the nucleus of M31 and the bulge at $r=23^{\prime \prime}$, have used the relation $\mathrm{Mgb} \approx 15 \mathrm{Mg}_{2}$ (Worthey 1994), and from the equations of Tantalo et al. (1998) we have derived differences of the parameters: $\Delta[\mathrm{Mg} / \mathrm{Fe}]=+0.12, \Delta \log \left(Z / Z_{\odot}\right)=+0.53$ and $\Delta \log t=-0.52$. This means that the magnesium-to-iron ratios are close and the nucleus is three times more metal-rich and three times younger than the bulge at $23^{\prime \prime}$ from the center. As for absolute values, the model of Tantalo et al. (1998) which gives the best set for the index combination in the nucleus is $Z=0.05$ (or $2.5 Z_{\odot}$ ), $[\mathrm{Mg} / \mathrm{Fe}]=+0.3$ and $t=6-7$ billion years. Consequently, in the bulge the stellar population metallicity is slightly below the solar one, and its age is over 15 billion years.

As M31 is known to have two nuclei, it would be important to localize the chemically distinct entity more exactly. For this purpose we have used the longslit data from the $4.2 \mathrm{~m}$ WHT obtained under much better seeing conditions than our observations. Figure 2 presents the absorption-line index variations along the slit for $\mathrm{CaI}$ and $\mathrm{FeI}$ in the position angle P.A. $=55^{\circ}$, very close to the line connecting two nuclei of $\mathrm{M} 31\left(43^{\circ} \pm 1^{\circ}\right)$. Both profiles exhibit maxima shifted to the south-west with respect to the continuum maximum. The continuum profile looks asymmetric; a Gaussian analysis reveals the presence of two components, a point-like one at $r=0$ and an extended one $\left(F W H M=7^{\prime \prime}\right)$ at $r=-0$ ".4, which we identify with two M31 nuclei, P1 and P2. So it is P2 which is the chemically and age decoupled nucleus of M31. This result is not unexpected. King et al. (1995) obtained an image of M31 at $\lambda=1750 \AA$; they had found that $\mathrm{P} 2$ is brighter in the ultraviolet than $\mathrm{P} 1$, in contrast to the visible. They have interpreted this fact as evidence for P2 metallicity overabundance because there exists a correlation between UV excess and metallicity in elliptical galaxies.

Figure 3 presents morphological characteristics of the central part of M31. It is fully consistent with earlier photometric results (see CFHT data in the work of Bacon et al. 1994). But there are some details which were not discussed 


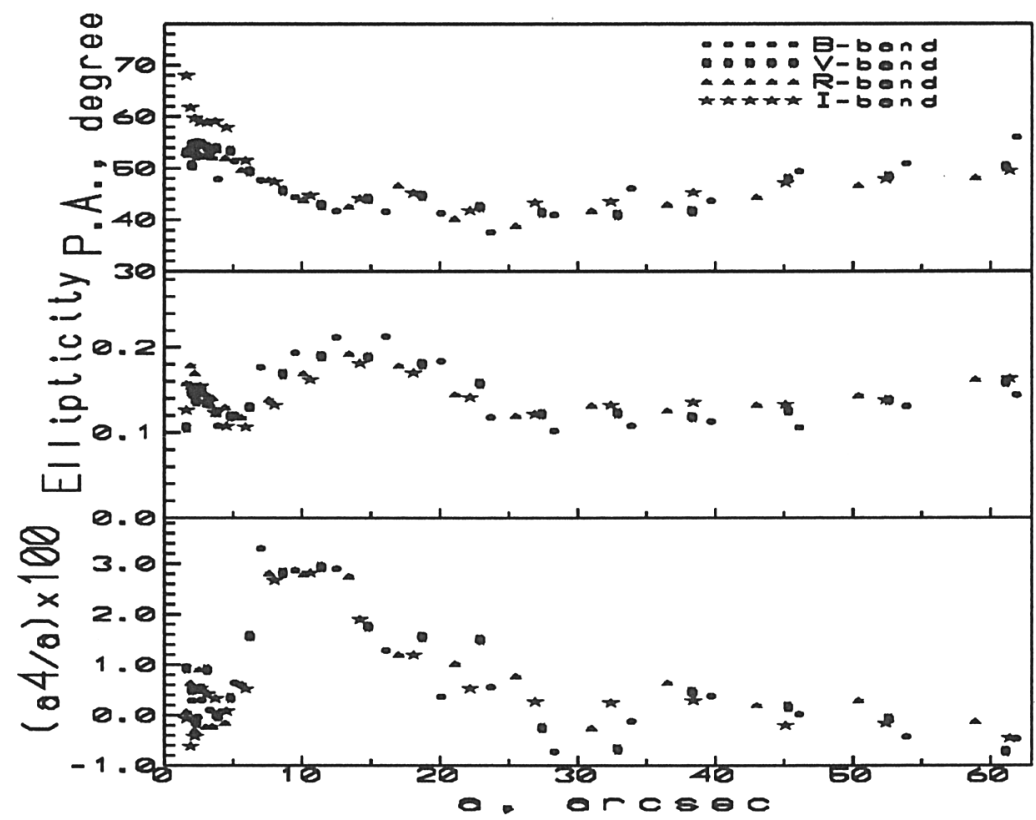

Figure 3. Radial variations of the isophote parameters Position Angle, ellipticity and $a_{4} / a$.

earlier. Over all the four spectral bands the major semi-axis range of $7^{\prime \prime}-25^{\prime \prime}$ is characterized by a local maximum of ellipticity and by a local minimum in P.A. which oscillates between $40^{\circ}$ and $44^{\circ}$, close enough to the orientation of the line of nodes of M31, P.A. $=38^{\circ}$. In the same radius interval $a_{4} / a$ is higher than $+1 \%$; outside, $a_{4} / a<+0.01$. This means that in the radius range of $7^{\prime \prime}-25^{\prime \prime}$ we see a stellar disk whose plane is close to the global plane of the galaxy. The central region of M31 is dominated by the bulge; at the distance of M31 the major semi-axis of $25^{\prime \prime}$ corresponds to a linear radius of $85 \mathrm{pc}$; therefore we are dealing with a so-called nuclear disk. Over the same radius range an emission line [OIII] $\lambda 5007$ is seen in our spectra (in the very center of M31, $r<5^{\prime \prime}$, emission lines are absent according to Bacon et al. 1994). We can conclude that the nuclear stellar disk of M31 contains also ionized gas.

\section{Discussion}

Earlier when chemically distinct nuclei were found in some elliptical galaxies, where they appeared to be resolved, a simultaneous discovery of circumnuclear stellar disks of comparable sizes allowed to match chemically distinct cores to the inner disks. Papers on NGC 4365 by Bender \& Surma (1992) and Surma \& Bender (1995) containing this conclusion are well known. We have also found two such cases, NGC 1052 and 4621 (Sil'chenko 1995, 1997). But in spiral galaxies the chemically distinct nuclei are unresolved. M31 is the first case when we are able to resolve the situation; it has destroyed our presumption: the inner disk 
detected from photometry has a radius of $80 \mathrm{pc}$, while the chemically distinct nucleus is less than 10 pc. Moreover, it is not $\mathrm{P} 1$ which is chemically distinct though only P1 is thought to be a giant stellar cluster (Emsellem \& Combes 1997), but P2 which is assumed to be a supermassive black hole the stellar content around which is still unknown. What may be the nature, and consequently the origin and structure, of the unresolved chemically distinct stellar nucleus in M31? The gaseous component of the inner disk seems to be unstable: we see spiral miniarms and local emission-line splitting, the emission-line intensity ratios evidence for a shock mechanism of gas excitation. So there must be radial gas inflow into the center of the galaxy resulting from momentum losses in the shock fronts. Perhaps, the gas accumulating in the center of M31 has fuelled a secondary star formation burst several billion years ago, which has left a chemically enriched nucleus. There exists still another explanation: if in the proximity of the supermassive black hole located inside P2 stars have some unusual structure, say, if their external atmospheres are stripped by tidal effects, then we see their hotter inner layers which are enriched by nucleosynthesis products. In this case the nucleus P2 may look like a chemically decoupled young nucleus too.

Acknowledgements. This research has made use of the La Palma Archive. WHT and JKT are operated on the island of La Palma by the Royal Greenwich Observatory in the Spanish Observatorio del Roque de los Muchachos of the Instituto de Astrofisica de Canarias. The work is supported by the grant of the Russian Foundation for Basic Researches 98-02-16196 and by the Russian State Scientific-Technical Program "Astronomy. Basic Space Researches" (section "Astronomy").

\section{References}

Bacon, R., Emsellem, E., Monnet, G., Nieto, J.L. 1994, A\&A, 281, 691

Bender, R. Surma, P. 1992, A\&A, 258, 250

Bica, E., Alloin, D., Schmidt, A.A. 1990, A\&A, 228, 23

Cohen, J.G. 1979, ApJ, 228, 405

Davidge, T.J. 1997, AJ, 113, 985

Davidge, T.J., Rigaut, F., Doyon, R., Crampton, D. 1997, AJ, 113, 2094

Dressler, A., Richstone, D.O. 1988, ApJ, 324, 701

Emsellem, E., Combes, F. 1997, A\&A, 323, 674

Joly, M., Andrillat, Y. 1973, A\&A, 26, 95

King, I.R., Stanford, S.A., Crane, Ph. 1995, AJ, 109, 164

Kormendy, J. 1988, ApJ, 325, 128

Lauer, T.R., Faber, S.M., Groth, E.J., Shaya, E.J., et al. 1993, AJ, 106, 1436

McClure, R.D. 1969, AJ, 74, 50

Morton, D.C., Andereck, C.D. 1976, ApJ, 205, 356

Sil'chenko, O.K. 1995, Pis'ma v Astron. Zh., 21, 323

Sil'chenko, O.K. 1996, Pis'ma v Astron. Zh., 22, 124

Sil'chenko, O.K. 1997, Astron. Zh., 74, 643 
Sil'chenko, O.K., Vlasyuk, V.V., Burenkov, A.N. 1997, A\&A, 326, 941 Spinrad, H., Liebert J.W. 1975, ApJ, 200, 582

Surma, P., Bender, R. 1995, A\&A, 298, 405

Tantalo, R., Chiosi, C., Bressan, A. 1998, A\&A, 333, 419

Tremaine, S. 1995, AJ, 110, 628

Vlasyuk, V.V. 1993, Astrofiz. issled. (Izv. SAO RAS), 36, 107

Worthey, G. 1994, ApJS, 95, 107 